\title{
Area-preserving dynamics of a long slender finger by curvature: a test case for the globally conserved phase ordering
}

\author{
Avner Peleg, Baruch Meerson, and Arkady Vilenkin \\ Racah Institute of Physics, Hebrew University of Jerusalem, Jerusalem 91904, Israel \\ Massimo Conti \\ Dipartimento di Matematica e Fisica, Universitá di Camerino, and Istituto Nazionale di Fisica della Materia, 62032, \\ Camerino, Italy
}

\begin{abstract}
A long and slender finger can serve as a simple "test bed" for different phase ordering models. In this work, the globally-conserved, interface-controlled dynamics of a long finger is investigated, analytically and numerically, in two dimensions. An important limit is considered when the finger dynamics are reducible to the area-preserving motion by curvature. A free boundary problem for the finger shape is formulated. An asymptotic perturbation theory is developed that uses the finger aspect ratio as a small parameter. The leading-order approximation is a modification of "the Mullins finger" (a well-known analytic solution) which width is allowed to slowly vary with time. This time dependence is described, in the leading order, by an exponential law with the characteristic time proportional to the (constant) finger area. The subleading terms of the asymptotic theory are also calculated. Finally, the finger dynamics is investigated numerically, employing the Ginzburg-Landau equation with a global conservation law. The theory is in a very good agreement with the numerical solution.
\end{abstract}

PACS numbers: $64.75 .+\mathrm{g}, 05.70 . \mathrm{Np}$, 05.70.Ln

\section{INTRODUCTION}

This work is motivated by the recent developments in phase-ordering theory. Phase ordering is the growth of order from disorder via domain growth and coarsening. As phase ordering systems are strongly nonlinear and highly disordered, their theoretical description remains challenging 11. To get insight, it is often useful to consider the coarsening dynamics of simple test objects. One such object is a spherical droplet of the "minority" phase. It serves as the building block of simplified phase-ordering theories for locally conserved (bulk-diffusion-controlled) 2] and globally conserved (interface-controlled) [3] systems.

Being too a simple object, a spherical droplet may not give sufficient insight. For example, it's dynamics (shape-preserving shrinking or expansion) look the same in both locally, and globally conserved systems. On the other hand, there are important differences in the phase-ordering behavior of locally and globally conserved systems. In both cases, an ensemble of droplets exhibits Ostwald ripening, and the corresponding meanfield theories, due to Lifshitz and Slyozov [2] and Wagner [3], respectively, predict dynamic scaling behavior of the droplet size distribution (with different dynamic exponents). However, for finite volume fractions, the difference in the type of conservation law leads to a different role of correlations. Correlations between neighboring droplets are much more important in the locallyconserved systems [- 4 , than in the globally-conserved ones [5]. Even bigger differences have been found in the phase ordering dynamics of locally and globally con- served systems with long-range correlations. Coarsening of fractal clusters shows dynamic scale invariance and "normal" scaling in the case of global conservation [6, and breakdown of scale invariance and anomalous scaling in locally conserved systems [7, 8 .

It is natural to attribute these differences to a basic difference in the character of transport: in globally conserved systems the transport is uninhibited by Laplacian screening effects, typical for locally conserved diffusioncontrolled systems. To study this basic difference in a simple setting, the dynamics of a single long and slender finger is often considered. It has been found recently [8] that, in the locally-conserved, diffusion-controlled system, the finger acquires a dumbbell shape and shows non-trivial dynamic scalings for the finger length and the "ball" size, while the initial finger width remains almost constant until a late stage of the dynamics. Furthermore, a finger-shaped domain (a "needle") served as a test object in still another conserved coarsening system: the one controlled by edge diffusion [9]. Finger-like objects appear naturally in two-dimensional simulations of dewetting [10], etc. In this work we investigate the globally conserved dynamics of a long slender finger. An additional motivation for studying the finger dynamics is a recent observation that, at a late stage of coarsening of fractal clusters, the cluster morphology shows long branches, or fingers, in both locally [7, 8, 11], and globally [6,12] conserved systems.

Here is an outline of the rest of the paper. In Section 2 we briefly review the Ginzburg-Landau equation with a global conservation law (a phase field model for globallyconserved phase ordering) and its general sharp interface 
formulation in two dimensions. Under a certain condition (that will be elucidated) this formulation is reduced to the area-preserving dynamics by curvature. In Section 3 we formulate a moving boundary problem for the finger dynamics and develop a perturbation theory which enables us to obtain an intermediate asymptotic solution for the finger shape. In Section 4 we return to the globally-conserved Ginzburg-Landau equation and investigate the finger dynamics numerically. Section 5 includes a brief discussion.

\section{GLOBALLY CONSERVED PHASE ORDERING: A PHASE FIELD MODEL AND ITS SHARP-INTERFACE LIMIT}

Globally-conserved phase ordering can be described by a simple phase field model 13 15. This model represents the dynamics in terms of a simple gradient descent

$$
\frac{\partial u}{\partial t}=-\frac{\delta F}{\delta u}
$$

with the Ginzburg-Landau free energy functional

$$
F[u]=\int\left[(1 / 2)(\nabla u)^{2}+V(u)+H u\right] d \mathbf{r}
$$

and a double-well potential $V(u)=(1 / 4)\left(1-u^{2}\right)^{2}$. The effective "magnetic field" $H=H(t)$ varies in time so as to impose global conservation law

$$
<u(\mathbf{r}, t)>=\frac{1}{L_{x} L_{y}} \int u(\mathbf{r}, t) d \mathbf{r}=\text { const } .
$$

Here $u(\mathbf{r}, t)$ is the order parameter field, $L_{x} \gg 1$ and $L_{y} \gg 1$ are the linear dimensions of the system (a twodimensional rectangular box), and the integration is carried out over the entire box. Eqs. (11)-(3) yield the nonlocal Ginzburg-Landau equation

$$
\frac{\partial u}{\partial t}=\nabla^{2} u+u-u^{3}-<u-u^{3}>.
$$

Either no-flux, or periodic boundary conditions are assumed.

For a wide class of initial conditions this coarsening system segregates, at late times, into large domains of "phase 1" and "phase 2" separated by thin domain walls (which width is of order unity) [5, 13, 16]. Correspondingly, a sharp interface theory can be developed for these late times [5]. At these times the magnetic field $H(t)$ is already small, $H(t) \ll 1$, and slowly varies with time. The phase field in the phases 1 and 2 is almost uniform and rapidly adjusts to the current value of $H(t)$, so that $u=-1-H(t) / 2$ and $1-H(t) / 2$, respectively. The normal velocity of the interface is given by [5]

$$
v_{n}(s, t)=\kappa(s, t)-\frac{3}{\sqrt{2}} H(t),
$$

where $\kappa$ is the local curvature and $s$ is the coordinate along the interface. The positive sign of $v_{n}$ corresponds to the interface moving towards phase 1 , while $\kappa$ is positive when the interface is convex toward phase 2 .

The dynamics of $H(t)$ is determined by [5]

$$
\dot{H}(t)=\frac{4 \Lambda(t)}{L_{x} L_{y}}\left(\overline{\kappa(s, t)}-\frac{3}{\sqrt{2}} H(t)\right),
$$

where

$$
\overline{\kappa(s, t)}=\frac{1}{\Lambda(t)} \oint \kappa(s, t) d s,
$$

and $\Lambda(t)$ is the total interface length. Equations (5) and (6) make a complete set and provide a general sharpinterface formulation to this problem. In some cases this formulation can be further simplified [5, 13]. Let us compute the rate of change of the domain area, $\dot{A}$ :

$$
\dot{A}(t)=\oint v_{n}(s, t) d s=\Lambda(t)\left[\overline{\kappa(s, t)}-\frac{3}{\sqrt{2}} H(t)\right] .
$$

If the two terms in the right hand side of Eq. (8) balance each other,

$$
H(t) \simeq \frac{\sqrt{2}}{3} \overline{\kappa(s, t)},
$$

the domain area remains approximately constant. Then, using Eqs. (5) and (9), we obtain

$$
v_{n}(s, t)=\kappa(s, t)-\overline{\kappa(s, t)} .
$$

Model (10) is known as area-preserving flow by curvature [17]. Dynamics (10) reduce the interface length of the system [13,17, leaving the areas of each of the phases constant. The only stable steady state of Eq. (10) (not attached to the boundaries of the system) is a single perfectly circular domain [13]. Under what condition is model (10) a good approximation to the more general sharp-interface model (5) and (6)? Consider Eq. (6) which includes the same combination $\bar{\kappa}-(3 / \sqrt{2}) H(t)$. Let us treat the term in the right hand side of Eq. (6) perturbatively, putting $H(t)=H^{(0)}(t)+h(t)$, where $H^{(0)}(t)=(\sqrt{2} / 3) \bar{\kappa}$ is the leading term, and $h(t)$ is a subleading term. Keeping terms up to order $h(t)$ we obtain:

$$
h(t)=-\frac{\sqrt{2} L_{x} L_{y}}{12 \Lambda} \dot{H}^{(0)}(t)=-\frac{L_{x} L_{y}}{18 \Lambda} \dot{\kappa}
$$

If there is only one (single-connected) domain, $\bar{\kappa}=$ $-2 \pi / \Lambda$. Therefore,

$$
h(t)=-\frac{\pi L_{x} L_{y}}{9} \frac{\dot{\Lambda}}{\Lambda^{3}} .
$$

Requiring $|h(t)| \ll\left|H^{(0)}(t)\right|$, we obtain the "areapreservation" criterion 


$$
\frac{L_{x} L_{y}|\dot{\Lambda}|}{\Lambda^{2}} \ll 1 .
$$

In Section 3 we will investigate the area-preserving dynamics of a long slender finger, and verify criterion (13) a posteriori.

\section{AREA-PRESERVING FINGER DYNAMICS: A PERTURBATION THEORY}

Let the initial condition for dynamics (10) represent a long and narrow rectangular bar with length $2 a_{0}$ and width $2 \Delta_{0}$. We will see that, with time, the bar evolves into a finger-shaped object. We place the origin of a Cartesian coordinate system in the finger's center and denote by $x$ the coordinate along the finger and by $y=$ $y(x, t)$ the instantaneous location of the finger boundary. Because of the symmetry with respect to the $x$-axis, we will be interested only in the upper boundary dynamics: $y(x, t) \geq 0$. In terms of $y(x, t)$ the local curvature is given by [18]:

$$
\kappa(s, t)=\frac{y_{x x}}{\left(1+y_{x}^{2}\right)^{3 / 2}},
$$

where $y_{x} \equiv \partial y(x, t) / \partial x$. The average curvature $\overline{\kappa(s, t)}$ is

$$
\overline{\kappa(s, t)}=\frac{-2 \pi}{\Lambda}=\frac{-\pi}{2 \int_{0}^{a(t)}\left(1+y_{x}^{2}\right)^{1 / 2} d x},
$$

where $a(t)$ is the time-dependent position of the finger tip. Since

$$
\frac{\partial y}{\partial t}=\left(1+y_{x}^{2}\right)^{1 / 2} v_{n},
$$

dynamics (10) imply the following evolution equation for $y(x, t)$ :

$$
\frac{\partial y}{\partial t}=\frac{y_{x x}}{1+y_{x}^{2}}+\frac{\pi\left(1+y_{x}^{2}\right)^{1 / 2}}{2 \int_{0}^{a(t)}\left(1+y_{x}^{2}\right)^{1 / 2} d x} .
$$

$a(t)$ is defined by the boundary conditions $y(x=a, t)=0$ and $y_{x}(x=a, t)=-\infty$. An additional boundary condition follows from the symmetry with respect to the $y$-axis: $y_{x}(x=0, t)=0$. Equation (14), a nonlinear integrodifferential equation in partial derivatives, describes a free-boundary problem. It is easy to check that the dynamics (14) preserve the finger area $A$.

It can be proved 13] that any convex domain evolving by Eq. (10) [and therefore by Eq. (14)] finally becomes a circle with the same area $A$. We are interested in the finger dynamics at times much smaller than those needed for approaching this equilibrium (but still large enough so that details of the initial shape are forgotten). The corresponding strong double inequality will be presented at the end of this Section.
As long as the ratio of the finger width to its length is small, we can use it as the small parameter of our theory. Indeed, near the finger tip the first (local) term in the right hand side of Eq. (14) is of the order of the inverse finger width, while the second (nonlocal) term is of the order of the inverse finger length. Therefore, the nonlocal term can be treated perturbatively. The unperturbed equation,

$$
\frac{\partial y}{\partial t}=\frac{y_{x x}}{1+y_{x}^{2}},
$$

was obtained more than 40 years ago by Mullins in the context of motion of grain boundaries [19]. It corresponds to a non-conserved motion by curvature: $v_{n}(s, t)=\kappa$. We notice immediately that the perturbed problem, Eq. (14), possesses an integral of motion (the finger area) which is absent in the corresponding unperturbed problem. This situation is unusual: more often than not perturbations destroy integrals of motion pertinent to the unperturbed system. This unusual property will be exploited in the following, as we will require area conservation in each order of the perturbation expansion.

Mullins [19] obtained a one-parameter family of traveling-wave solutions of Eq. (15) describing a halfinfinite constant-width finger retreating by its tip's curvature:

$$
y=Y(x, t)=\frac{1}{c} \arccos [\exp (-c \xi)],
$$

where $\xi=a(t)-x, a(t)=a_{0}-c t$ is the time-dependent position of the finger tip, and the constant speed of retreat $c$ is the parameter of this family of solutions. The finger's half-width $\Delta=\pi /(2 c)$ is constant. We will show that this solution (with two important modifications) can serve as the leading (or zero) order approximation of our perturbation theory for the area-preserving finger dynamics. First, we introduce a (slow) time-dependence in the parameter $c$. Second, we "stick together" two identical (very long but finite) half-fingers. The resulting ansatz is the following:

$$
Y(x, t)=\frac{1}{c(t)} \arccos [\exp (-c(t) \xi)],
$$

where $\xi=a(t)-|x|$, and $a(t)>0$ is yet unknown. The finger half-width $\Delta(t)=\pi / 2 c(t)$ is now time-dependent; its growth with time makes up, in the area conservation, for the finger shortening. As a function of $x$, ansatz (17) is continuous everywhere. Its $x$-derivative is not continuous at $x=0$. However, as far as $\epsilon(t)=\Delta(t) / a(t)$ is very small: $\epsilon(t) \ll 1$, the $x$-derivatives at $x=0$ from left and right are of order of $\exp (-1 / \epsilon)$, that is exponentially small. This and other exponentially small effects will be neglected throughout the paper. Because of the symmetry with respect to the $y$-axis, we will consider only the region $0 \leq x \leq a(t)$. The perturbation expansion for $y(x, t)$ is 


$$
y(x, t)=Y(x, t)+\delta y(x, t)+\ldots,
$$

where $\delta y(x, t)$ is the subleading (or first order) term. Inserting Eq. (117) into Eq. (14) and keeping only the zero order terms, we obtain

$$
\dot{a}=-c=-\pi /(2 \Delta) .
$$

The evolution equation for $\Delta(t)$ follows from the requirement of area conservation in the zero order. Calculating the area under the $Y(x, t)$ profile, we obtain:

$$
\int_{0}^{a(t)} Y(x, t) d x=a \Delta-\frac{2 \Delta^{2} \ln 2}{\pi}+O(\exp (-1 / \epsilon)) .
$$

One can see that the finger area in the zero order is simply $A=4 a \Delta$. Demanding $\dot{A}=0$ and using Eq. (19), we obtain:

$$
\dot{\Delta}=\pi /(2 a) .
$$

We will see later that the same equation follows from the analysis of the first order correction. The solution of the zero order equations (19) and (21) is

$$
a^{(0)}(t)=a_{0} \exp \left(-\pi t / 2 a_{0} \Delta_{0}\right),
$$

and

$$
\Delta^{(0)}(t)=\Delta_{0} \exp \left(\pi t / 2 a_{0} \Delta_{0}\right) .
$$

Therefore, in the zero order, the finger shortens (and its width grows) exponentially with time. The characteristic growth time is of order of the finger area. The zero order solution yields a criterion for the validity of the perturbation theory. The aspect ratio of the evolving finger should be very small which leads to

$$
t \ll a_{0} \Delta_{0} \ln \frac{a_{0}}{\Delta_{0}},
$$

This condition sets an upper limit for the times for which our perturbation expansion is valid.

We now turn to calculating the small correction $\delta y(x, t)$. Since the nonlocal term of Eq. (14) is already of order $\epsilon$, we evaluate it on the zero-order solution $Y(x, t)$ which yields $\pi(2 a)^{-1}\left(1+Y_{x}^{2}\right)^{1 / 2}$. Introduce a new variable $u$ :

$$
u(x, t)=\exp (-c(t) \xi) .
$$

Substituting Eq. (18) into Eq. (14) and linearizing, we get a linear partial differential equation for $\delta y(x, t)$ :

$$
\begin{aligned}
\delta y_{t}= & \left(1-u^{2}\right) \delta y_{x x}-2 c u^{2} \delta y_{x} \\
& -\frac{(c+\dot{a}) u}{\left(1-u^{2}\right)^{1 / 2}}+\frac{\dot{c}}{c^{2}} q(u)+\frac{\pi}{2 a\left(1-u^{2}\right)^{1 / 2}},
\end{aligned}
$$

where

$$
q(u)=\frac{u \ln u}{\left(1-u^{2}\right)^{1 / 2}}+\arccos u .
$$

It is convenient to go over to new independent variables $\xi$ and $t$. We define $\delta y(x, t)=f(\xi, t)$ and obtain $\delta y_{t}=f_{t}+\dot{a} f_{\xi}$. As the $t$-dependence in the new variables is slow, the term $f_{t}$ can be neglected in this order of the perturbation scheme. Therefore, we are left with an ordinary differential equation (the slow time enters as a parameter):

$$
\begin{aligned}
\left(1-u^{2}\right) f_{\xi \xi}+c\left(2 u^{2}+1\right) f_{\xi}= & \frac{(c+\dot{a}) u}{\left(1-u^{2}\right)^{1 / 2}}-\frac{\dot{c}}{c^{2}} q(u) \\
& -\frac{\pi}{2 a\left(1-u^{2}\right)^{1 / 2}}
\end{aligned}
$$

Going over from $\xi$ to $u$ and defining $f(\xi)=g(u)$ we obtain

$$
\begin{aligned}
\left(1-u^{2}\right) g_{u u}-3 u g_{u}= & \frac{(c+\dot{a})}{c^{2}} \frac{1}{u\left(1-u^{2}\right)^{1 / 2}}-\frac{\dot{c}}{c^{4}} \frac{q(u)}{u^{2}} \\
& -\frac{\pi}{2 a c^{2} u^{2}\left(1-u^{2}\right)^{1 / 2}} .
\end{aligned}
$$

Define $\Phi(u)=g_{u}(u)$. The general solution of the homogeneous equation for $\Phi(u)$ is $C \Phi_{0}(u)$, where

$$
\Phi_{0}(u)=\left(1-u^{2}\right)^{-3 / 2},
$$

and $C$ is an arbitrary constant. Therefore, we look for the general solution of Eq. (29) in the form $\Phi(u)=$ $C(u) \Phi_{0}(u)$, where $C(u)$ is an unknown function. Substituting this into Eq. (29) and integrating, we obtain

$$
C(u)=\frac{(c+\dot{a})}{c^{2}} \ln u-\frac{\dot{c}}{c^{4}} s(u)+\frac{\pi}{2 a c^{2} u}+\alpha(t),
$$

where

$$
\begin{aligned}
s(u)= & \frac{1}{2} \ln ^{2} u+\frac{1}{2} \arccos ^{2} u-\ln u- \\
& \frac{\left(1-u^{2}\right)^{1 / 2} \arccos u}{u},
\end{aligned}
$$

and $\alpha(t)$ is an arbitrary function of time. Going back to $g(u)$, we can write the first order correction to the finger shape as

$$
g(u)=\int_{0}^{u} C(u) \Phi_{0}(u) d u+\beta(t),
$$

where $\beta(t)$ is another arbitrary function of time. The boundary conditions for $g(u)$ are $g(0)=0$ and $g(1)=$ 0 . The first boundary condition gives $\beta(t)=0$, while the second boundary condition should be used to find an equation for $\dot{a}$ in the first order. Before doing this, an analysis of the integrals in Eq. (33) should be made. To prevent divergence of $g(u)$ at $u=1$ we must require 
$\alpha(t)=-\pi /(2 c a)$. Similarly, to prevent divergence of $g(u)$ at $u=0$ we must require $\dot{c} / c^{2}=-1 / a$. The latter equation is equivalent to Eq. (21) obtained in the leading order from the requirement of area conservation.

The equation for $\dot{a}$ in the first order of the perturbation theory is obtained from the boundary condition $g(1)=0$ which requires calculating the integral in Eq. (33) from $u=0$ to $u=1$. This calculation yields

$$
\dot{a}=-c+\frac{3 \ln 2}{a}=-\frac{\pi}{2 \Delta}+\frac{3 \ln 2}{a} .
$$

It is now possible to obtain an explicit expression for $g(u)$. An integration from 0 to $u$ in Eq. 33 gives:

$$
\begin{aligned}
g(u)= & \frac{1}{c^{2} a}\left[w_{1}(u)-\arcsin u \ln \left(\frac{8 u}{e}\right)+\right. \\
& \left.\frac{\pi}{2} \ln \left(\frac{2}{e\left[1+\left(1-u^{2}\right)^{1 / 2}\right]}\right)+w_{2}(u)\right],
\end{aligned}
$$

where

$$
w_{1}(u)=\frac{2 u \ln u \ln \left(8 u^{1 / 2} / e\right)+u \arccos ^{2} u+\pi(1-u)}{2\left(1-u^{2}\right)^{1 / 2}}
$$

and

$$
w_{2}(u)=2 \int_{0}^{u} \frac{\arcsin u}{u} d u
$$

In the absence of a conservation law, we would have to go to the second order of the perturbation scheme to obtain the equation for $\dot{c}$ in the first order. In our areapreserving perturbation scheme this equation can be obtained already in the first order. Notice that the firstorder correction obeys the following conservation law:

$$
\frac{d}{d t} \int_{0}^{a(t)} \delta y(x, t) d x=\dot{a}[f(\xi=a, t)-f(\xi=0, t)]=0
$$

where the last equality results from the boundary conditions. This relation combined with the requirement that the finger area is preserved leads to

$$
\frac{d}{d t} \int_{0}^{a(t)} Y(x, t) d x=0
$$

It should be stressed that functions $a(t)$ and $\Delta(t)$ entering Eq. (39) now include first order corrections. Now, using Eqs. (20), (34) and (39) and keeping terms only up to the first order in $\epsilon$, we obtain

$$
\dot{\Delta}=\frac{\pi}{2 a}-\frac{\Delta \ln 2}{a^{2}} .
$$

Equations (34) and (40) are the equations for $\dot{a}$ and for $\dot{\Delta}$ in the first order of the perturbation scheme. This set of equations is autonomous and therefore integrable. In the framework of the perturbation theory it is more consistent to solve the equations perturbatively by linearization. To do this, we exploit the conservation law (39) which, together with Eq. (20), allows us to express $\Delta(t)$ as a function of $a(t)$ or vice versa. The expression obtained is then linearized with respect to $\Delta / a$ and inserted into Eq. (34) (or (40)). This yields the following equations:

$$
\begin{gathered}
\dot{a}=-\frac{\pi a}{2 a_{0} \Delta_{0}}+\frac{4 \ln 2}{a}-\frac{a \ln 2}{a_{0}^{2}}, \\
\dot{\Delta}=\frac{\pi \Delta}{2 a_{0} \Delta_{0}}+\frac{\Delta \ln 2}{a_{0}^{2}}-\frac{\Delta \ln 2}{a^{2}}-\frac{\Delta^{3} \ln 2}{a_{0}^{2} \Delta_{0}^{2}} .
\end{gathered}
$$

We are looking for solutions in the form of $a(t)=a^{(0)}(t)+$ $a^{(1)}(t)$ and $\Delta(t)=\Delta^{(0)}(t)+\Delta^{(1)}(t)$, where $a^{(1)}(t)$ and $\Delta^{(1)}(t)$ are first order corrections. Solving the resulting linear equations, we obtain:

$$
\begin{gathered}
a^{(1)}(t)=\frac{8 \Delta_{0} \ln 2}{\pi} \sinh \left(\frac{\pi t}{2 a_{0} \Delta_{0}}\right)- \\
\frac{t \ln 2}{a_{0}} \exp \left(-\frac{\pi t}{2 a_{0} \Delta_{0}}\right) \\
\Delta^{(1)}(t)=-\frac{4 \Delta_{0}^{2} \ln 2}{\pi a_{0}} \exp \left(\frac{\pi t}{a_{0} \Delta_{0}}\right) \sinh \left(\frac{\pi t}{2 a_{0} \Delta_{0}}\right)+ \\
\frac{\Delta_{0} t \ln 2}{a_{0}^{2}} \exp \left(\frac{\pi t}{2 a_{0} \Delta_{0}}\right)
\end{gathered}
$$

We checked that when solving Eqs.(34) and (40) numerically, the results differ from the approximate analytical results (43) and (44) by less than 1\%. Finally, requiring $a^{(0)}(t) \gg a^{(1)}(t)$ and $\Delta^{(0)}(t) \gg \Delta^{(1)}(t)$ and using (22), (23), (43), and (44), one can see that the linearization procedure is valid for times obeying the same strong inequality (24). Therefore, the linearization procedure is consistent with the perturbation theory.

The validity of our perturbative solution is limited by not too long times, see inequality (24). On the other hand, one should wait until irrelevant details of the initial bar shape are forgotten, and the solution approaches $Y(x, t)$. Typically, it takes time needed for the bar tip to pass the distance of order of the characteristic tip size $1 / c \sim \Delta$. The tip speed is of order $1 / \Delta$, so the "waiting time" of the theory can be estimated as $\Delta_{0}^{2}$. Our perturbative solution represents, therefore, an intermediate asymptotics, valid for

$$
\Delta_{0}^{2} \ll t \ll a_{0} \Delta_{0} \ln \frac{a_{0}}{\Delta_{0}} .
$$

Finally, we should check the area-preservation criterion (13). In the leading order we have: $\Lambda(t) \sim a^{(0)}(t)$ and 
$\dot{\Lambda}(t) \sim \dot{a}^{(0)}(t) \sim 1 / \Delta^{(0)}(t)$. Using (22) and (23) in (13) we obtain:

$$
t \ll a_{0} \Delta_{0} \ln \left(\tilde{f} a_{0}\right),
$$

where $\tilde{f}=\left(a_{0} \Delta_{0}\right) /\left(L_{x} L_{y}\right)$ is the area fraction occupied by the finger.

\section{NUMERICAL SOLUTION}

In order to test the predictions of the perturbation theory we performed numerical simulations with the nonlo-

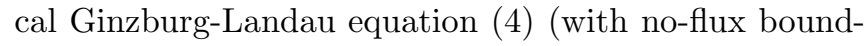
ary conditions), taking a long and narrow rectangular bar as the initial condition. We used an explicit Euler integration scheme to advance the solution in time, and second order central differences to discretize the Laplace operator. A mesh size of $\Delta x=\Delta y=0.5$ was found sufficient for an accurate resolution of the interface. A time step of $\Delta t=0.05$ was required for numerical stability. The bar width $2 \Delta_{0}=50$ was chosen to guarantee area conservation with a good accuracy [see Eq. (46)]. The simulation was carried out up to a time $t_{f}=9 \cdot 10^{4}$ which was long enough to distinguish between an exponential and linear dynamic behavior for $a(t)$ and $\Delta(t)$. The bar length $2 a_{0}=8 \cdot 10^{3}$ was chosen, so that the parameter $\epsilon(t)=\Delta(t) / a(t)$ was sufficiently small even at the end of simulation (when it reached 0.1). The bar was placed in the center of a rectangular box $8020 \times 220$. Because of symmetry with respect to the $x$ and $y$ axes only the quadrant $x>0, y>0$ was actually simulated. We checked that the finger area was conserved with an accuracy better than $1 \%$.

The time dependence of the finger half-length $a(t)$ obtained from the numerical simulations is shown in Fig. 1. The same graph also shows the theoretical values of $a(t)$ in the zeroth order $\left[a^{(0)}(t)\right.$, Eq. (22)], and first order $\left[a^{(0)}(t)+a^{(1)}(t)\right.$, Eq. (43)] of the perturbation theory. A good agreement between the numerical and analytical results is obtained already in the zeroth order. This agreement is improved further by the first order correction. For example, at the final time of the simulation $t_{f}=9 \cdot 10^{4}$, there is a $9 \%$ difference between the numerical value of $a(t)$ and $a^{(0)}(t)$, and only a $1.4 \%$ difference between the numerical value of $a(t)$ and $a^{(0)}(t)+a^{(1)}(t)$.

The numerically found half-width of the finger $\Delta(t)$ is shown in Fig. 2. Its comparison with the zero-order values $\Delta^{(0)}(t)$ [Eq. (23)] and with the first-order values $\Delta^{(0)}(t)+\Delta^{(1)}(t)[$ Eq. (44)] is also shown. Again, there is a good agreement already in the zeroth order, and this agreement is further improved by the first order correction. At the final time of the simulation, the numerical value of $\Delta(t)$ differs by $6 \%$ from $\Delta^{(0)}(t)$, and by less than $2 \%$ from $\Delta^{(0)}(t)+\Delta^{(1)}(t)$.

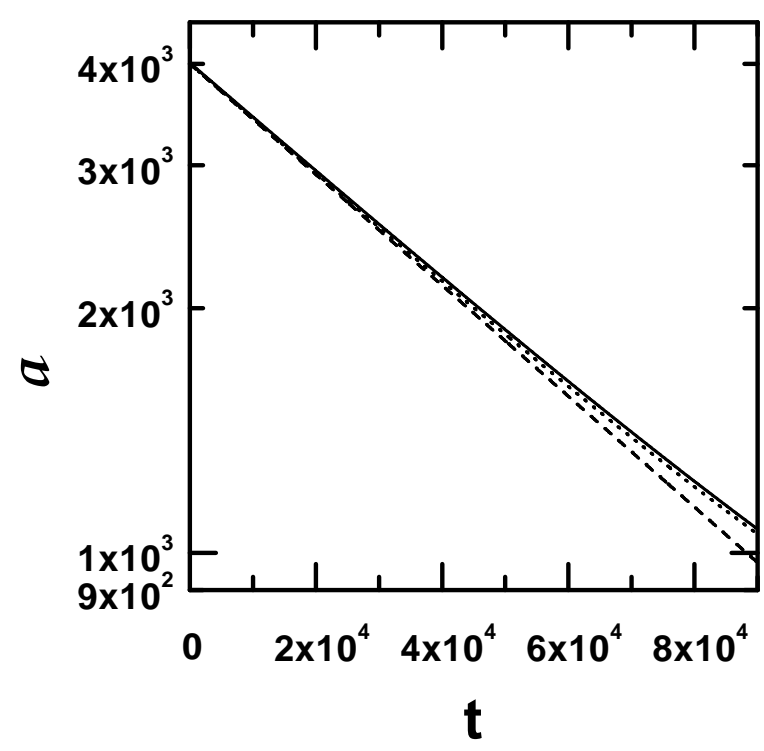

FIG. 1. A semi-log plot of the finger half-length $a$ versus time. The solid line represents $a(t)$ found numerically. The dashed line shows the zero-order analytic result $a^{(0)}(t)$. The dotted line is the first-order analytic result $a^{(0)}(t)+a^{(1)}(t)$.

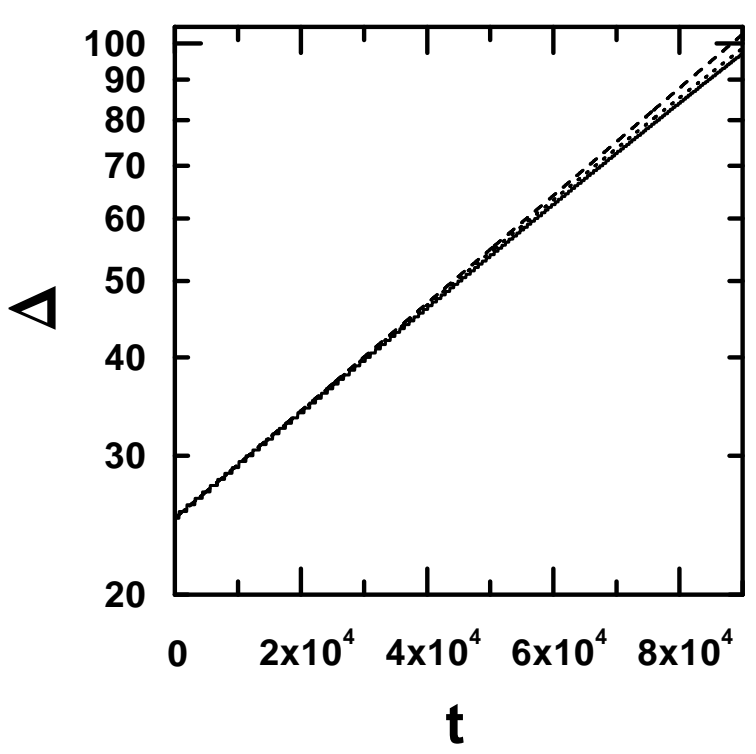

FIG. 2. A semi-log plot of the finger half-width $\Delta$ versus time. The solid line is $\Delta(t)$ found numerically. The dashed line shows the zero-order analytic result $\Delta^{(0)}(t)$. The dotted line is the first-order analytic result $\Delta^{(0)}(t)+\Delta^{(1)}(t)$.

Fig. 3 shows the finger shape $y(x, t)$ found numerically, at the final time of the simulation $t_{f}=9 \cdot 10^{4}$ (notice the different scales in the $x$ and $y$ axes). Also shown are the following zero- and first order theoretical results evaluated at $t=t_{f}$ : 


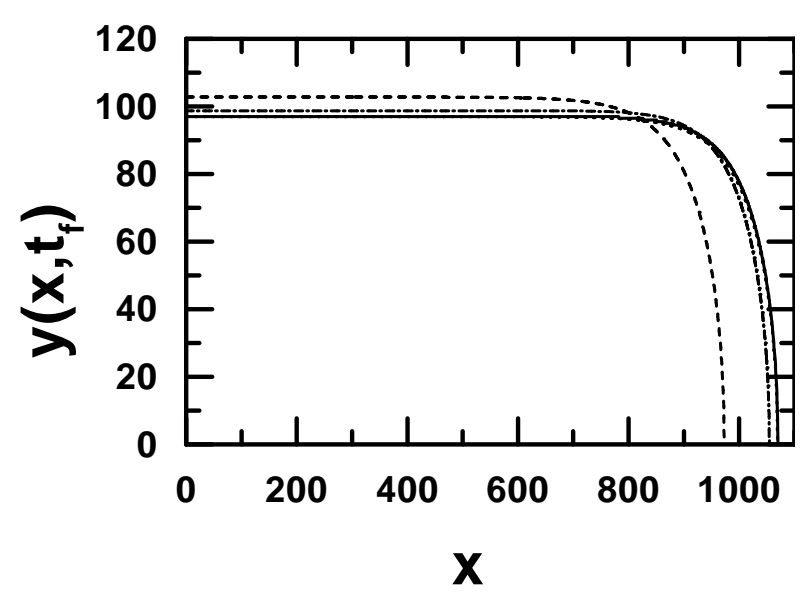

FIG. 3. The finger shape $y\left(x, t_{f}\right)$ (solid line). The dashed line is the function $Y^{(0)}\left(x, t_{f}\right)$ defined by Eq. (47), the dashed-dotted line is the function (48), and the dotted line is the function (49). All the functions are evaluated at $t=t_{f}=9 \cdot 10^{4}$. The dotted line is indistinguishable from the solid line.

$$
Y^{(0)}(x, t)=\frac{1}{c^{(0)}(t)} \arccos \left[\exp \left(-c^{(0)}(t) \xi^{(0)}\right)\right]
$$

where $\xi^{(0)}=a^{(0)}(t)-x$, and

$$
y_{s}(x, t)=\frac{1}{c_{s}(t)} \arccos \left[\exp \left(-c_{s}(t) \xi_{s}\right)\right]+\delta y(x, t)
$$

where $\xi_{s}=a_{s}(t)-x, a_{s}=a^{(0)}+a^{(1)}$, and $c_{s}=c^{(0)}+c^{(1)}$. In addition, shown is the function $Y_{\text {num }}(x, t)$ defined by:

$Y_{\text {num }}(x, t)=\frac{1}{c_{\text {num }}(t)} \arccos \left[\exp \left(-c_{\text {num }}(t) \xi_{\text {num }}\right)\right]$,

where $\xi_{\text {num }}=a_{\text {num }}(t)-x$, and $a_{\text {num }}(t)$ and $c_{\text {num }}(t)$ are the numerical values of $a(t)$ and $c(t)$, respectively. The function $Y_{\text {num }}(x, t)$ is also evaluated at $t=t_{f}$. The comparison with the zero-order prediction $Y^{(0)}\left(x, t_{f}\right)$ shows a good agreement even at this relatively late time, when $\Delta / a \simeq 0.1$. The agreement is further improved by the first-order theory. The comparison with $Y_{\text {num }}\left(x, t_{f}\right)$ shows an excellent agreement, implying that the subleading term $\delta y(x, t)$ is very small compared to the leading term $Y(x, t)$. Fig. 4 which shows the theoretical result for $\delta y(x, t)$ at $t=t_{f}$, calculated by using Eqs. (35)(37), confirms the later conclusion. We should add that a very good agreement was found between the theoretical $\delta y(x, t)$ and the numerical $\delta y(x, t)$, which is obtained by subtracting $Y_{\text {num }}(x, t)$ from the numerical result for $y(x, t)$.

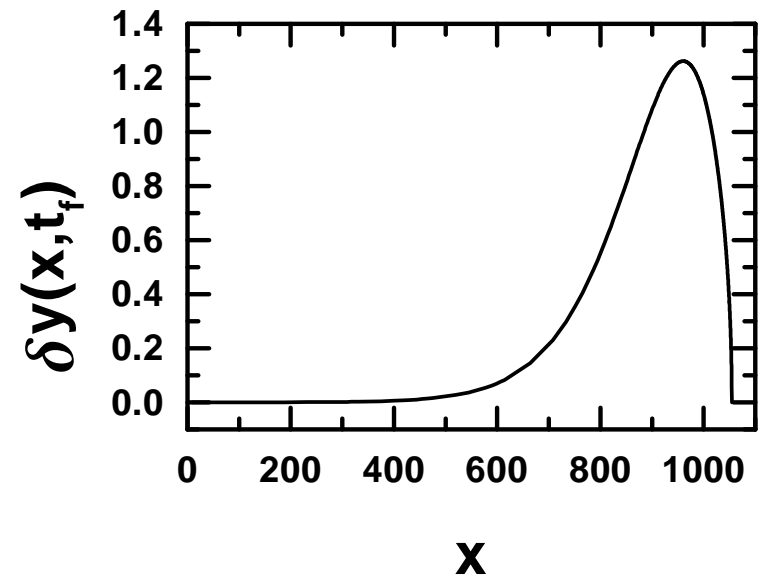

FIG. 4. The subleading term $\delta y\left(x, t_{f}\right)$ at the final time of the simulation, as obtained by the perturbation theory [Eqs. (35)-(37)].

\section{CONCLUSIONS}

We investigated the dynamics of a long slender fingershaped domain undergoing globally-conserved (interfacecontrolled) coarsening. We worked in the parameter region where these dynamics can be reduced to areapreserving motion by curvature. The nonlinear moving boundary problem for the finger shape was solved by using an asymptotic perturbation theory which employed, as the zero-order solution, a straightforward modification of the Mullins solution for a finger retreating by the curvature of its tip. Both the leading, and the subleading terms of the solution were calculated analytically and verified by a numerical solution of the corresponding phase-field equation: the Ginzburg-Landau equation with a global conservation law.

The coarsening dynamics of the finger look quite differently from those observed in the case of a locally conserved (diffusion-controlled) system [8]. The finger preserves its simple shape, while its characteristic length and width are changing on the same time scale. Therefore, the global character of transport (uninhibited by Laplacian screening effects characteristic of the locally conserved system) manifests itself already in the simple setting of finger dynamics.

\section{ACKNOWLEDGEMENTS}

This work was supported in part by the Israel Science Foundation, administered by the Israel Academy of Sciences and Humanities. 
[1] A.J. Bray, Adv. Phys. 43, 357 (1994).

[2] I.M. Lifshitz and V.V. Slyozov, J. Phys. Chem. Solids 19, 35 (1961).

[3] C. Wagner, Z. Electrochem. 65, 581 (1961).

[4] M. Marder, Phys. Rev. A 36, 858 (1987).

[5] B. Meerson and P.V. Sasorov, Phys. Rev. E 53, 3491 (1996).

[6] A. Peleg, M. Conti, and B. Meerson, cond-mat/0007220.

[7] M. Conti, B. Meerson, and P.V. Sasorov, Phys. Rev. Lett. 80, 4693 (1998).

[8] M. Conti, B. Meerson, and P.V. Sasorov, e-print condmat/9912426.

[9] R. Thouy, N. Olivi-Tran, and R. Jullien, Phys. Rev. B 56, 5321 (1997).

[10] J. Koplik and J.R. Banavar, Phys. Rev. Lett. 84, 4401 (2000).

[11] T. Irisawa, M. Uwaha and Y. Saito, Europhys. Lett. 30, 139 (1995).

[12] T. Irisawa, M. Uwaha and Y. Saito, Fractals 4, 251 (1996).

[13] J. Rubinstein and P. Sternberg, IMA J. Appl. Math. 48, 249 (1992).

[14] C. Sire and S.N. Majumdar, Phys. Rev. E 52, 244 (1995).

[15] A.D. Rutenberg, Phys. Rev. E 54, 972 (1996).

[16] A.S. Mikhailov, Foundations of Synergetics. 1. Distributed Active Systems (Springer-Verlag, Berlin, 1993).

[17] M. Gage, Contemp. Math. 51, 51 (1986).

[18] R. Courant, Differential and Integral Calculus, Vol. I (Interscience Publishers, New York, 1956).

[19] W.W. Mullins, J. Appl. Phys. 27, 900 (1956). 